\title{
Caracterización de las acumulaciones loéssicas en el valle medio del río Tajo, España
}

\section{Characterization of loess accumulations in the middle Tajo river valley, Central Spain}

\author{
R. García ${ }^{1}$, J.A. González², M.D. Petit ${ }^{3}$, M.I. Rucandio ${ }^{4}$
}

\begin{abstract}
RESUMEN
Las llanuras del centro de España muestran un gran interés paleoclimático. Se sitúan en torno a los $40^{\circ}$ de latitud Norte y entre 450-650 metros de altitud. Durante el Pleistoceno, sus condiciones sedimentarias se han desarrollado bajo climas mediterráneos, degradados o separados por pequeños hiatos. En estas llanuras, el loess es una de las acumulaciones superficiales más adecuadas para la reconstrucción de ambientes pasados y para la determinación de cambios climáticos. Se han analizado, para el presente estudio, más de un centenar de muestras en estos depósitos eólicos, en el que la determinación de las propiedades físicas se ha llevado a cabo por medio de análisis mecánicos. La edad del loess ha sido obtenida por termoluminiscencia y se puede asociar a las fases isotópicas del oxígeno (OIS) 3 y 2 (período Würm y tardiglaciar). Los loess están correlacionados con otras formaciones frías de estos ambientes y, entre ellas, con los depósitos estratificados sobre las laderas en relación con intensos fríos y bruscos cambios térmicos y estacionales.
\end{abstract}

Palabras clave: loess, Pleistoceno, Río Tajo, sedimentología.

\begin{abstract}
The Central Spain plains have a great paleoclimatic interest. They are located around $40^{\circ} \mathrm{N}$ and at heights of $450 / 650$ m.a.s.l. During the Pleistocene their paleosedimentary environment developed under Mediterranean climates, degraded or separated by small hiatus. In these plains, loess represents one of the most suitable surface deposits to reconstruct past environments and for the determination of climate changes. For this study, over one hundred samples from such deposits were collected, in which the determination of physical properties was carried out through mechanical analysis. The age of the loess has been obtained by thermoluminescence and can be linked to the Oxygen isotope (OIS) 3 and 2 (Würm period and Late Glacial) phases. The loess are correlated with other cold features in this area. Among them, are the «stratified debris slope deposits» related to breakdown by seasonal freezing and thawing.
\end{abstract}

Key words: loess, Pleistocene, Tajo River, sedimentology.

\section{Introducción}

Desde mediados del siglo Xx se han estudiado distintos tipos de acumulaciones loéssicas en las regiones mediterráneas, países balcánicos (Brunnacker, 1969b) e Italia (Cremaschi, 1987). También hay referencias de loess en varias zonas de la Península Ibérica, como en Andalucía (Brunnacker, 1969a;
Brunnacker \& Lözek, 1969), Cataluña (Solé et al., 1957), País Valenciano (Dumas, 1979). Sin embargo, en los mapas de distribución de loess mundiales (Flint, 1971; Haase et al., 2007) España no aparece, ni siquiera como un «posible depósito». Esto podría ser debido al carácter descriptivo empleado hasta ahora y aplicado en la identificación de estas formaciones eólicas, así como a la casi total ausencia de

\footnotetext{
1 Dpto. Geología y Geoquímica. Facultad de Ciencias. Universidad Autónoma. 28049 Madrid. España. Email: rosario.garcia@uam.es

2 Dpto. Geografía. Facultad de Filosofía y Letras. Universidad Autónoma. 28049 Madrid. España. Email: juanantonio.gonzalez@uam.es

${ }^{3}$ Dpto. Química Analítica. Facultad de Ciencias. Universidad Autónoma. 28049 Madrid. España. Email: mdolores.petit@uam.es

4 Unidad de espectroscopia. CIEMAT. 28040 Madrid. España. Email: isabel.rucandio@ ciemat.es
} 
datos sedimentológicos y mineralógicos, que permitan la confirmación sobre la existencia de un seguro depósitos de loess.

La mayoría de las acumulaciones loéssicas se relacionaron con sedimentos glaciares o formados a partir de la rotura de las rocas en procesos de hielo/deshielo. Más tarde, los materiales sueltos fueron trasladados por los fuertes vientos en latitudes medias y altas durante los períodos fríos del Pleistoceno. En la actualidad, la vinculación de los depósitos loéssicos con las regiones periglaciares es un hecho y el loess es el depósito de la Tierra más extendido en estas regiones, alrededor del 10\% de la superficie terrestre está cubierta por loess (Pye, 1987).

Las llanuras aluviales y los páramos calizos del centro de la Península Ibérica muestran un gran interés paleoclimático. Durante el Pleistoceno sus ambientes sedimentarios se desarrollaron en torno a climas mediterráneos, degradados o suspendidos por cortas fluctuaciones, en condiciones de frío extremo, sequedad y humedad mayores que las actuales. Todos estos acontecimientos han contribuido a cambios radicales en la evolución del paisaje. Por lo tanto, los indicios siguientes denotan climas específicos y procesos de acumulación de materiales: períodos cálidos y húmedos, laderas cubiertas de vegetación, taludes que disminuyen la erosión y procesos de disolución kárstica. En tiempos pretéritos la precipitación de carbonatos generó una gran cantidad de depósitos tobáceos a lo largo de los valles y sus laderas. Mediante isótopos de uranio se han datado las mayores acumulaciones tobáceas coincidiendo con edades 190-250ky, 90130ky, 30-ky 50ky y 10-4ky (Ordóñez et al., 1991).

No obstante, las condiciones semiáridas generan, además, costras calizas, sedimentos lacustres en la zonas de «playa» y depósitos eólicos. Por último, los climas fríos glaciales identificados en las montañas y con características periglaciares se reflejan en las zonas de menor altitud.

En el presente trabajo, se han estudiado las acumulaciones eólicas ubicadas en el sur y suroeste de Madrid (García, 2004), situadas en el valle del río Tajo, entre las ciudades de Toledo y Tarancón (fig. 1). El área se localiza entre 490 y $700 \mathrm{~m}$ de altitud en el centro de la cuenca sedimentaria de Madrid, donde yeso, marga, arcilla y caliza (Mioceno Medio y Superior) son las rocas más frecuentes. Durante el Cuaternario, el río Tajo y sus afluentes (Jarama, Henares y Tajuña) generaron amplios valles mordiendo materiales neógenos. Los materia- les loéssicos descritos en este trabajo tienen una composición mineralógica rica en carbonatos, cuarzo, feldespatos y yeso, este último en proporciones variables.

El valle del río Tajo presenta dos generaciones de depósitos eólicos, en torno a la isoyeta a $400 \mathrm{~mm}$ : la más antigua está compuesta por limos y arenas depositados, probablemente, durante el Pleistoceno Medio. La otra, del Pleistoceno reciente, está compuesta por sedimentos de grano fino (limo y arena fina) con arcillas. Estas características texturales son típicas de los depósitos de loess. Datos de campo muestran «true loess» o depósitos de loess verdaderos y «loess-like» aquellos que se les pueden asimilar. Los primeros han sido transportados por la acción del viento y exhiben un espesor no superior a 5 metros; se disponen en extensos mantos con orientación W-E y están compuestos por masivas acumulaciones, sin estratificación, escasos poros y son ricos en carbonatos.

Los segundos consisten en loess que luego fueron retomados por procesos de ladera y erosión. Por ello, frecuentemente ofrecen materiales finos dispuestos en lechos o estructuras sedimentarias generadas por esporádicos flujos de agua. Debido al carácter erosional de su superficie, paleosuelos y otras formaciones edáficas no pueden ser identificados en las partes altas de los perfiles. Las verdaderas acumulaciones loéssicas comprenden varias unidades geomorfológicas (terrazas, glacis, pequeños y asimétricos valles, depósitos coluviales) que se identifican con diferentes edades del Pleistoceno. Estas unidades geomorfológicas sugieren que la mayoría de estos depósitos eólicos son del Pleistoceno reciente (González et al., 2000). La figura 2 recoge algunas manifestaciones de los loess del valle del río Tajo estudiadas.

\section{Materiales y métodos}

Los trabajos de cartografía geomorfológica y toma de muestras se llevaron a cabo entre los años 1995 y 1998 asociados con los datos obtenidos sobre la distribución de loess desde la observación de fotografía aérea. La morfología y las estructuras internas de los loess se lograron tanto en afloramientos como en excavaciones artificiales.

Se han analizado 106 muestras de loess tanto true loess como loess-like, situadas en diferentes unidades geomorfológicas. Las muestras fueron descritas de acuerdo con las directrices de la FAO (FAO- 


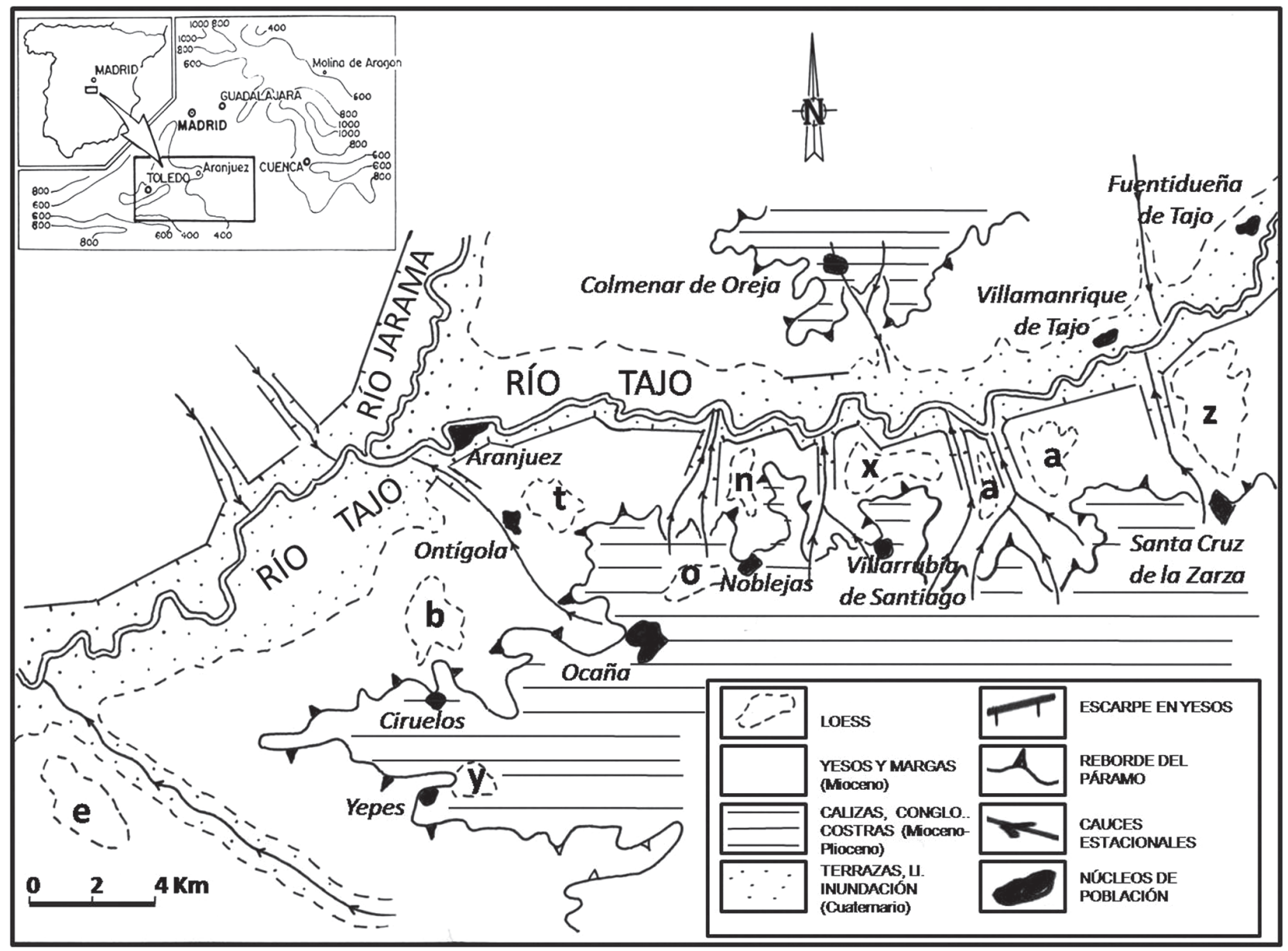

Fig. 1.-Mapa de situación para los depósitos loéssicos estudiados.

UNESCO 1977, 1990). Sus propiedades se han analizado siguiendo los métodos del Soil Survey Staff (1992). El color se ha establecido según la carta de colores Münsell.

La distribución del tamaño de partícula se determinó por el método de la pipeta después de eliminar la materia orgánica con $\mathrm{H}_{2} \mathrm{O}_{2}$ y dispersar agitando con hexametafosfato sódico (Loveland \& Whalley, 1991). El pH se midió potenciométricamente en una suspensión 1:2.5 suelo/agua con un pHmetro ORION SA 720 y la conductividad con conductimetro HANNA HA-8733.

La determinación de las propiedades físicas de las muestras analizadas en este estudio se ha llevado a cabo por medio de análisis mecánicos. Las curvas de frecuencia acumulativa se han realizado mediante separación mecánica (tamices) en los tamaños mayores y para las fracciones limo y arcilla por centrifugación.

\section{Discusión de resultados}

Teniendo en cuenta la localización espacial de los depósitos de loess, las muestras han sido clasificadas en nueve grupos, como puede verse en la tabla 1.

\section{Tabla 1.-Grupo de muestras seleccionadas y su localización}

\begin{tabular}{clcc}
\hline $\begin{array}{c}\text { Símbolo } \\
\text { del } \\
\text { grupo }\end{array}$ & $\begin{array}{c}\text { Área de campo } \\
\text { seleccionada }\end{array}$ & $\begin{array}{c}\text { № de muestras } \\
\text { correspondientes } \\
\text { a true loess }\end{array}$ & $\begin{array}{c}\text { № de muestras } \\
\text { correspondientes } \\
\text { a loess-like }\end{array}$ \\
\hline a & Arroyo Viloria & 2 & 14 \\
b & Ciruelos & 4 & 1 \\
e & Villamejor & 2 & 5 \\
n & Noblejas & 10 & 2 \\
o & Mesa de Ocaña & 0 & 6 \\
t & Ontígola & 11 & 0 \\
x & Villarrubia de Santiago & 15 & 13 \\
y & Yepes & 4 & 0 \\
Z & Santa Cruz de la Zarza & 8 & 9 \\
\hline
\end{tabular}




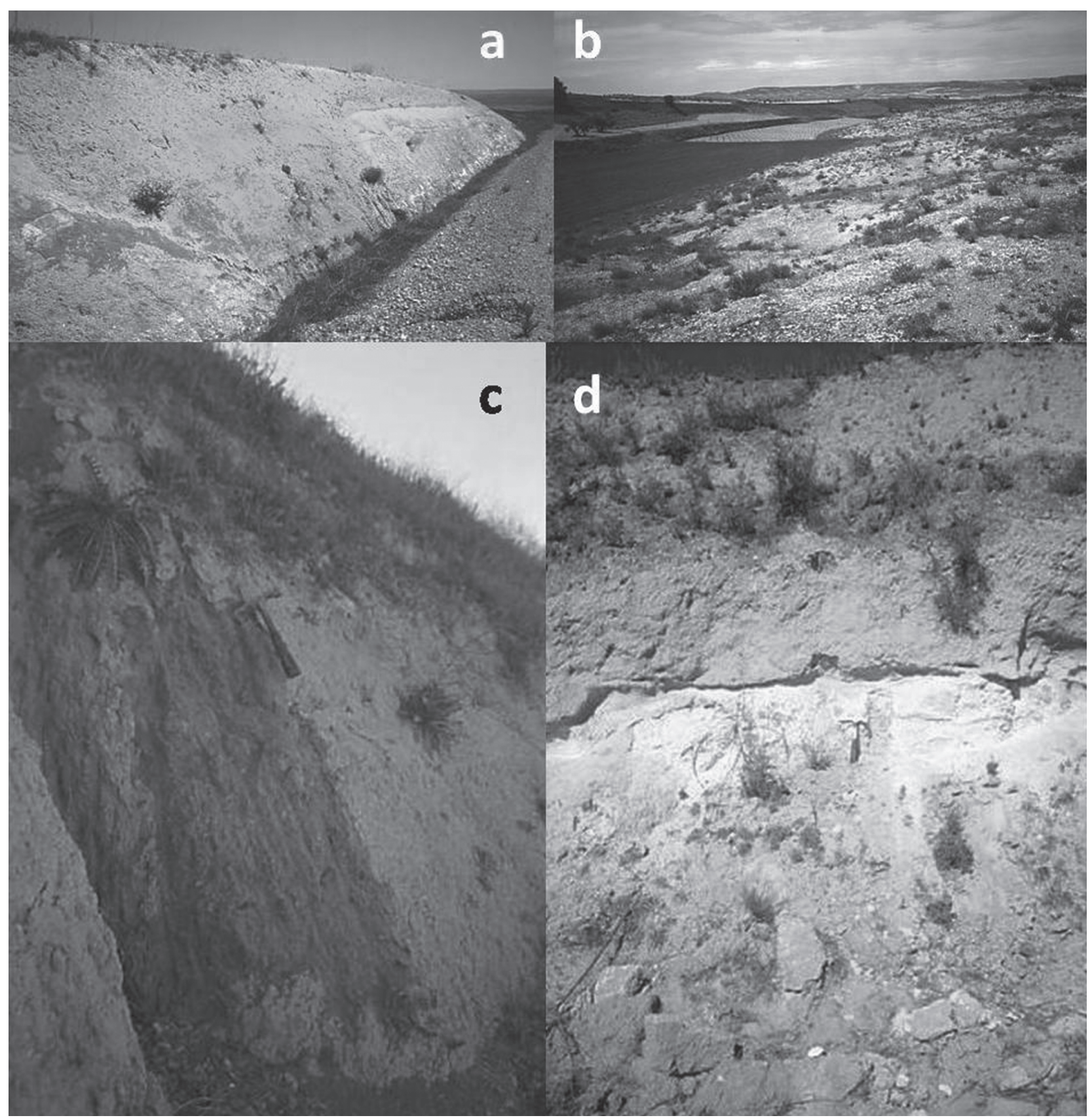

Fig. 2.-a) True loess fosilizando un vallecito en la margen derecha del río Tajo. b) Valle asimétrico en la margen izquierda del río Tajo, al fondo loess y en primer plano, el sustrato de yesos. c) True loess en la Mesa de Ocaña. $d$ ) True loess sobre las margas del Mioceno.

\section{Color}

En líneas generales, y atendiendo a las características morfológicas de los materiales loéssicos, se puede decir que se trata de muestras de color 7.5 YR 7/6 (amarillo rojizo) según la Münsell Color Chart, sin estructura o débilmente estructurados, sueltos en seco y sin poros ni rocas.

\section{Características granulométricas}

Dentro de los nueve grupos mencionados antes se han considerado los true loess y los loess-like, ambos manifiestan acumulaciones masivas sin estructuras internas. Teniendo en cuenta la granulometría (figs. 3 y 4) se puede decir que todas las muestras son predominantemente de textura limoarcillosa (grupos b, e, 


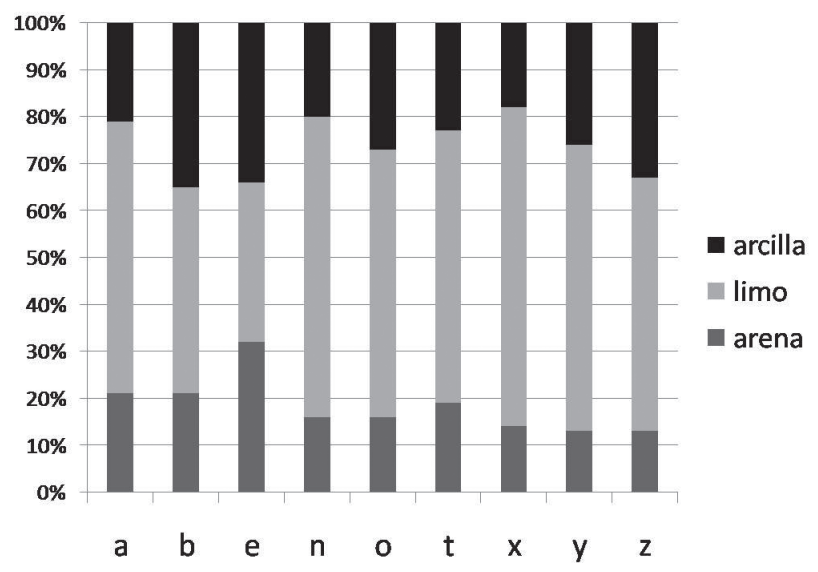

Fig. 3.-Diagrama de barras que recoge las proporciones entre las fracciones arena/limo/arcilla (a: Arroyo Viloria; b: Ciruelos; e: Villamejor; n: Noblejas; o: Mesa de Ocaña; t: Ontígola; x: Villarrubia de Santiago; y: Yepes; z: Santa Cruz de la Zarza).

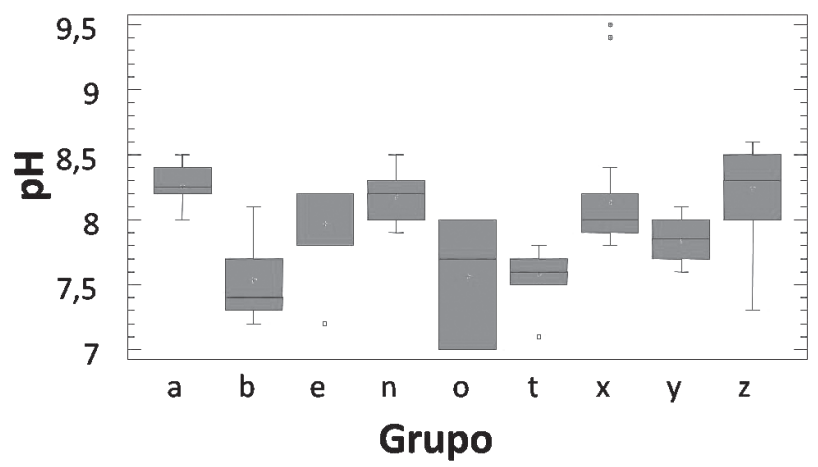

Fig. 5.-Gráfico de Box \& Whiskers para valores de pH (a: Arroyo Viloria; b: Ciruelos; e: Villamejor; n: Noblejas; o: Mesa de Ocaña; t: Ontígola; x: Villarrubia de Santiago; y: Yepes; z: Santa Cruz de la Zarza).

n, o, x, y, z), arcillosa (grupo a) y limosa fina (grupo t). Según Popov (1972), los loess están constituidos por materiales en polvo $(0,001-0,05 \mathrm{~mm})$ con o sin estratificación y compuestos por partículas finas de diámetro medio entre 0,005 y $0,05 \mathrm{~mm}$ (Morozov, 1962). Es importante destacar que todos los depósitos de la Mesa de Ocaña (grupo o) corresponden a loess-like y que las muestras recogidas en Ontígola ( $\mathrm{t}$ ) y Yepes (y) son todas true loess.

\section{Valores de $\mathrm{pH}$}

Los valores de $\mathrm{pH}$ se representan utilizando los gráficos box \& whiskers, un histograma, como método de ayuda a la interpretación en la distribución de datos. En estos dibujos (figs. 5 y 6), cada

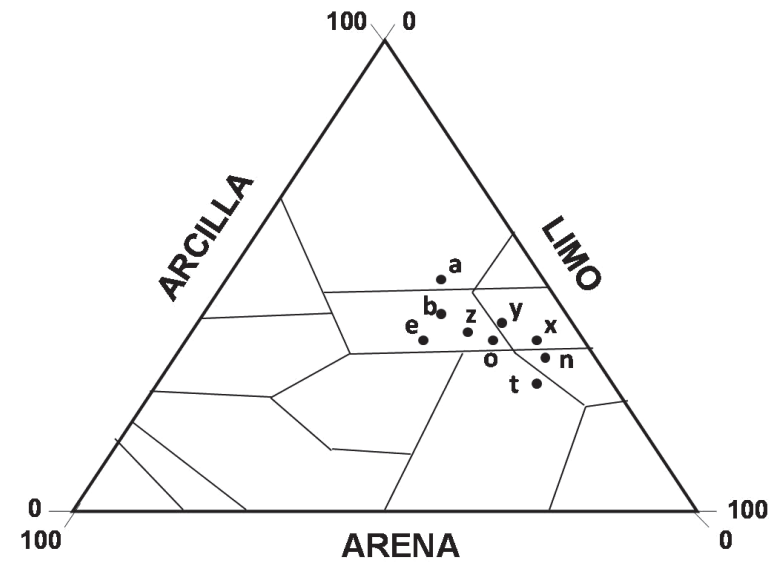

Fig. 4.-Relaciones granulométricas en los nueve grupos de muestras (a: Arroyo Viloria; b: Ciruelos; e: Villamejor; n: Noblejas; o: Mesa de Ocaña; t: Ontígola; x: Villarrubia de Santiago; y: Yepes; z: Santa Cruz de la Zarza).
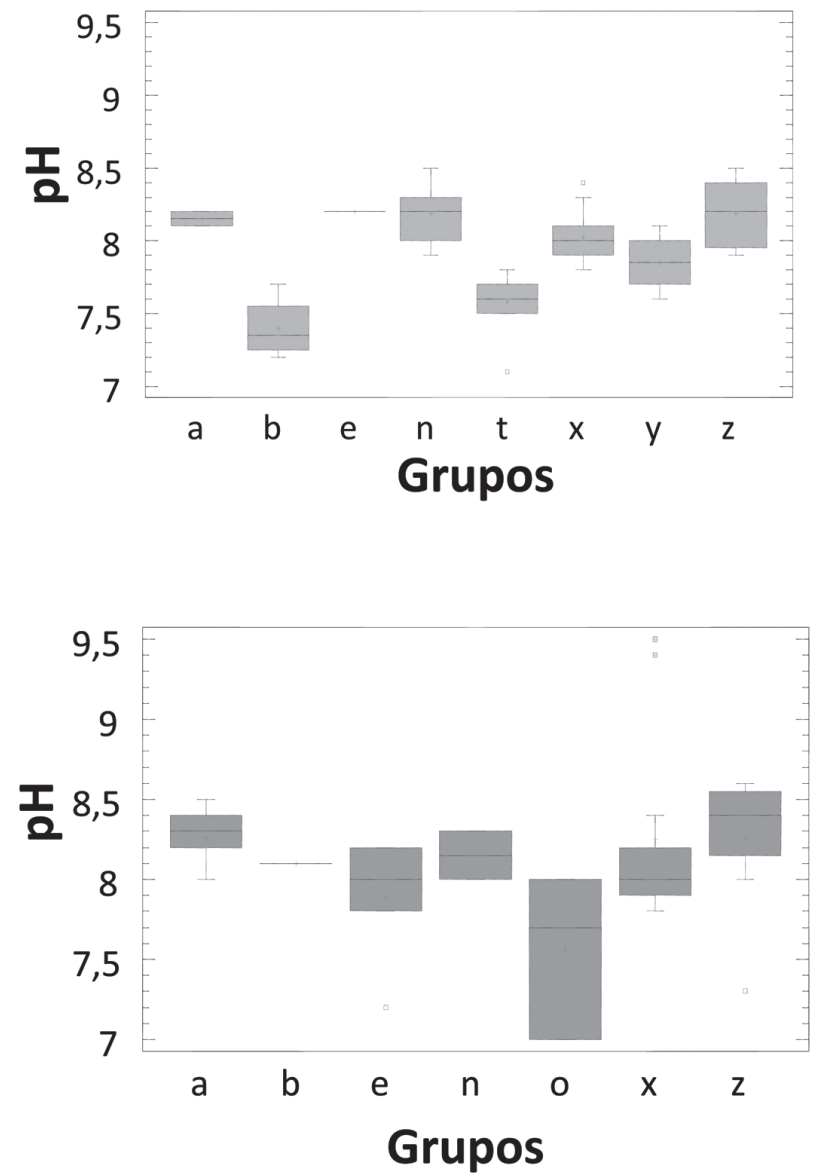

Fig. 6.-Arriba) Gráfico de Box \& Whiskers sobre valores de $\mathrm{pH}$ en true loess. Abajo) Gráfico de Box \& Whiskers sobre valores de $\mathrm{pH}$ en loess-like (a: Arroyo Viloria; b: Ciruelos; e: Villamejor; n: Noblejas; o: Mesa de Ocaña; t: Ontígola; x: Villarrubia de Santiago; y: Yepes; z: Santa Cruz de la Zarza). 
caja representa un grupo de datos donde en la mitad se delinea la media (línea horizontal dentro de la caja). Las líneas verticales que se prolongan desde cada extremo de la caja (whiskers) aportan los datos de los cuartiles 1,5. Dentro de las cajas, correspondiendo con el tercer intercuartil e identificados con puntos rojos, se definen los datos sospechosos.

Todas las muestras analizadas tienen $\mathrm{pH}$ alcalino, con valores comprendidos entre 7,5 y 8,5 (fig. 5) con algunos valores anómalos en muestras de Ciruelos (grupo b) y Mesa de Ocaña (grupo o). Estos valores de $\mathrm{pH}$ son similares a los descritos por Qizhong \& Honghan (1988) en loess del Norte de China, coincidiendo con las condiciones oxidantes de los entornos alcalinos.

Teniendo en cuenta los datos obtenidos en la medida de $\mathrm{pH}$ en los true loess (fig. 6, arriba), el promedio de los valores es muy similar y homogéneo (entre 7,7 y 8,2), con la excepción de las muestras procedentes de Ciruelos (grupo b). En este gráfico se puede observar que los loess del Arroyo Viloria (grupo a) tienen el $\mathrm{pH}$ más alto y la menor dispersión. La figura 6 abajo representa el gráfico para valores de $\mathrm{pH}$ en loess-like, donde los loess de Ciruelos (grupo b) tienen valores de $\mathrm{pH}$ superiores a sus respectivos grupos en los true loess; los valores de $\mathrm{pH}$ son muy similares para los grupos $\mathrm{e}, \mathrm{n}, \mathrm{x}$ y z en los loess verdaderos (true loess) y loess-like presentando la mayor dispersión en el grupo de los tomados en la Mesa de Ocaña (grupo o).

\section{Valores de conductividad}

Los valores de conductividad (fig. 7) están representados en un gráfico de Box \& Whiskers en donde se reconoce una gran variabilidad, con excepción de las muestras de Noblejas (grupo n). Considerando todos los grupos establecidos, el valor promedio se sitúa cerca de $1 \mathrm{dS} / \mathrm{m}$. La mayor dispersión en los valores de conductividad se produce en la Mesa de Ocaña (grupo o), Ontígola (grupo t), Villamejor (grupo e) y Yepes (grupo y). Las muestras del grupo x (Villarrubia de Santiago) muestran los valores máximo y mínimo.

\section{Conclusiones}

La región semiárida del Valle Medio del río Tajo Medio se caracteriza por sus extensas acumulaciones de loess.

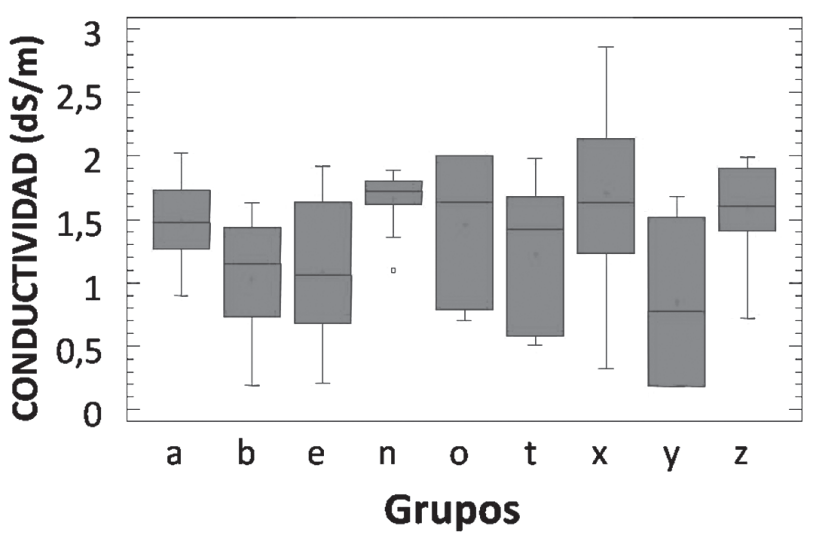

Fig. 7.-Gráfico de Box \& Whiskers para los valores de conductividad (a: Arroyo Viloria; b: Ciruelos; e: Villamejor; n: Noblejas; o: Mesa de Ocaña; t: Ontígola; x: Villarrubia de Santiago; y: Yepes; z: Santa Cruz de la Zarza).

Características sedimentológicas y geomorfológicas, establecidas en nuestro estudio, permiten identificar estas formaciones eólicas como acumulaciones tipo loess (true loess y loess-like).

La edad de los loess puede estar asociada con la OIS 3 y 2 (período Würm y tardiglaciar) y son correlativos con otras formaciones frías características en este ámbito, entre ellas los depósitos estratificados de ladera. El limo loéssico es un producto originado durante la fragmentación de materiales en los procesos de hielo/deshielo y ayudan a comprender el papel de la interacción mutua en las condiciones paleoclimáticas, la morfología y el material rocoso con la alta susceptibilidad de los yesos en la roca madre.

Sin embargo, la mayor parte del material fino que compone los loess proviene de la deflación de los sedimentos aluviales vinculados a las terrazas de los ríos Tajo y Jarama. Muchos procesos pueden ser responsables de la génesis de loess. El papel de los ambientes climáticos fríos parece ser indispensable en la formación de los depósitos loéssicos, así como la acción del viento y la falta de cubiertas vegetales. Son demasiado importantes los efectos de la sequía, como sugiere la ubicación de los depósitos de loess en la zona que hoy recibe menos precipitaciones en el centro de España.

Las propiedades de los loess analizados están relacionadas con su ubicación geográfica. Los loess examinados varían de textura limosa a ricos en arena, dependiendo de la proximidad a la fuente original del limo y las distancias recorridas durante el transporte. Pueden ser fácilmente diferenciados los true loess y los loess-like por los valores de $\mathrm{pH}$. 
Los valles orientados en dirección sur-norte son los que colectan mejor los loess, sobre todo en valles asimétricos, todo relacionado con los vientos del oeste que condicionan la sedimentación.

\section{Referencias}

Brunnacker, K. (1969a). Observations en Espagne et en Grece, Supplement au Bulletin de l'Association Française pour l'étude du Quaternaire, 67-69.

Brunnacker, K. (1969b). Affleurements de loess dans les régions nord-méditerranées. Revue de Geographie Physique et de Geologie Dynamique, 2: 325-334.

Brunnacker, K. \& Lözek, V. (1969). Lössorkanmen in Südostspanien. Zeitschrift für Geomorphologie, 13: 297-316.

Cremaschi, M. (1987). Loess deposits of the Plain of the $P o$ and of the adjoining Adriatic Basin (Northern Italy). In: Loess and Periglacial Phenomena (Pecsi, M. \& French, H.M., eds.), Akademia Kiadó, Budapest, $125-140$.

Dumas, B. (1979). Variations climatiques quaternaires et morphogénèse dans le Sud du Levant espagnol. Mediterranée, 27-35.

FAO-UNESCO (1977). Guidelines for soil profile description. Rome, 66 pp.

FAO-UNESCO (1990). Soil map of the world. Revised legend. Soils Bulletin 60. Rome.

Flint, R.F. (1971). Glacial and Quaternary geology, New York, Wiley, $892 \mathrm{pp}$.

García, R. (2004). Loess y otras acumulaciones en el valle medio del río Tajo y en sus páramos calizos: Sector Aranjuez-Tarancón. Tesis Doctoral. Universidad Autónoma de Madrid, 963 pp.

González, J.A.; Asensio, I.; Fernández, A.; García, R.; González, M.J.; Guerrero, L. \& Rubio, V. (2000). Acumulaciones de origen frío en el modelado de los paisajes de la Rama castellana del Sistema Ibérico y de la Submeseta Sur. In: Procesos y formas periglaciares en la montaña mediterránea (Lozano Tena, M.V.; Peña Monné, J.L. \& Sánchez Fabre, M., eds.), Instituto de Estudios turolenses, Teruel, 149-160.

Haase, D.; Fink, J.; Haase, G.; Ruske, R.; Pecsi, M.; Richter, H.; Altermann, M. \& Jager, K.D. (2007). Loess in Europe - its spatial distribution based on a European Loess Map, scale 1:2.500.00. Quaternary Science Reviews, 26: 1301-1312.

Loveland, P.J. \& Whalley, W.R. (1991). Particle size analysis. In: Soil analyses: physical methods (Smith, K.A. \& Mullins, C.E., eds.), Marcel Dekker, New York, 271-328.

Morozov, S.S. (1962). The loess soils classification. Proceedings of the Meeting on the engineering properties of rocks and methods of their study, Ministerio de Construcción de Polonia, Varsovia, 12-18.

Ordóñez, S.; Calvo, J.P.; García del Cura, M.A.; Alonso Zarza, A.M. \& Hoyos, M. (1991). Sedimentology of sodium sulphate deposits and special clays from the Tertiary Madrid Basin (Spain). Special Publications of the International Association of Sedimentology, 13: 39-55.

Popov, A.I. (1972). Les loess et dépôts loessoïdes, produit des processus cryolithogènes. Biuletin Peryglaciarny, 21: 193-200.

Pye, K. (1987). Aeolian dust and dust deposits. Academic Press, Londres, 334 pp.

Qizhong, W. \& Honghan, Z. (1984). Paleoclimatic records in the loess sections of North Xinjiang, China, in Loess deposits of the South Island, New Zealand and the soils formed or them, New Zealand Journal of Geology and Geophysics, 7: 811-838.

SOIL SURVEY STAFF (1994). Keys to Soil Taxonomy, $6^{\text {th }}$ edition, SCS-USDA, Washington DC, 306 pp.

Solé Sabaris, L.; Porta, J.; Solé, N.; Cuerda, J.; Muntaner, A. \& Colom, G. (1957). Livret guide de l'excursion L: Levant et Majorque, 5ème, Congrès Intern., INQUA, Madrid-Barcelona, 53 pp.

Recibido el 3 de diciembre de 2009 Aceptado el 25 de febrero de 2010 Publicado online el 5 de mayo de 2010 